Slavica

bruxellensia

\section{Slavica bruxellensia}

Revue polyphonique de littérature, culture et histoire

slaves

$7 \mid 2011$

Les Slaves, le Midi et l'Orient

\title{
Le Politicien de Shawgi Badri : espoirs et désillusions d'un communiste soudanais à Prague
}

\author{
Xavier Luffin
}

\section{OpenEdition}

\section{Journals}

Édition électronique

URL : http://journals.openedition.org/slavica/867

DOI : $10.4000 /$ slavica. 867

ISSN : 2034-6395

Éditeur

Université libre de Bruxelles - ULB

\section{Référence électronique}

Xavier Luffın, «Le Politicien de Shawgi Badri : espoirs et désillusions d'un communiste soudanais à Prague », Slavica bruxellensia [En ligne], 7 | 2011, mis en ligne le 16 octobre 2011, consulté le 03 mai 2019. URL : http://journals.openedition.org/slavica/867 ; DOI : 10.4000/slavica.867

Ce document a été généré automatiquement le 3 mai 2019.

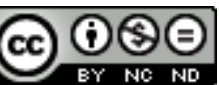

Les contenus de Slavica bruxellensia sont mis à disposition selon les termes de la Licence Creative Commons Attribution - Pas d'Utilisation Commerciale - Pas de Modification 3.0 France. 


\title{
Le Politicien de Shawgi Badri : espoirs et désillusions d'un communiste soudanais à Prague
}

\author{
Xavier Luffin
}

\section{L'auteur et son œuvre}

1 Shawgi Badri est un écrivain soudanais qui a publié deux recueils de nouvelles, Almushbak (La Pince à linge) ${ }^{1}$ et Hakâwî Umdurmân (Le Conteur d'Omdourman) ${ }^{2}$, et qui intervient régulièrement dans la presse soudanaise, qu'il s'agisse de quotidiens imprimés ou de sites littéraires et journalistiques sur la Toile.

2 L'auteur est né en 1945 et a grandi dans la ville d'Omdourman, à côté de Khartoum, mais il réside depuis quelques années en Suède. Ces deux éléments de sa biographie sont fondamentaux pour bien comprendre son œuvre car les personnages de ses nouvelles évoluent généralement dans l'un de ces deux univers - Omdourman et, secondairement, la Suède.

3 L'attachement de l'écrivain à la ville d'Omdourman va au-delà de l'amour d'un homme pour sa ville d'origine. En effet, pour les Soudanais, en particulier pour les musulmans du nord du pays, cette ville revêt une importance historique de premier plan, notamment parce que le Mahdi s'y était installé après avoir défait les troupes britanniques à Khartoum en $1885^{3}$. C'est dans cette ville aussi que se trouvent encore quelques édifices qui témoignent de cette période historique, comme par exemple le mausolée d'Al-Mahdî et la maison de son successeur, le calife 'Abdallah. 


\section{L'émigration dans la littérature soudanaise}

4 Le thème de l'émigration tient une place importante dans la littérature soudanaise, et ce pour au moins trois raisons. La première, parfois inconsciente, est l'influence fondamentale du roman Mawsim al-hijra ilâ al-shamâl (Saison d'une migration vers le nord) ${ }^{4}$, de l'écrivain Al-Tayyib Sâlih, sur ses compatriotes.

5 Ce roman, qui relate notamment la vie tumultueuse de Mustafa Sa'îd, un étudiant soudanais qui multiplie les aventures avec des femmes britanniques lors de son séjour à Londres, une sorte de revanche du colonisé sur le colonisateur, a profondément et durablement marqué les critiques et les chercheurs, tant dans le monde arabe que dans le monde occidental, en particulier dans les pays anglo-saxons, où il est souvent considéré comme l'un des romans-phares de la littérature postcoloniale ${ }^{5}$.

6 Hélas, le succès de ce roman a en quelque sorte éclipsé le restant de l'œuvre de Sâlih, auteur de plusieurs romans et de quelques recueils de nouvelles, mais aussi celle des autres écrivains soudanais, pourtant nombreux et souvent talentueux ${ }^{6}$.

7 La seconde raison est que le thème de l'émigration est devenu un lieu commun dans la littérature arabe contemporaine, qu'elle soit soudanaise, égyptienne, libanaise ou marocaine. Ceci est somme toute assez logique, puisque depuis la période des indépendances - et même avant en réalité, mais dans une moindre mesure - la plupart des pays arabes ont vu une partie importante de leur population émigrer en Europe ou ailleurs, tantôt pour des raisons économiques, tantôt pour des raisons politiques ou autres. Parmi les romans arabes abordant le thème de l'émigration, certains sont devenus des classiques, notamment 'Usfûr min al-sharq (L'Oiseau d'Orient)7 de Tawfîq Al-Hakîm, dont le héros est un jeune Égyptien qui se retrouve à Paris - mais chaque année sortent de nouveaux romans qui parlent de cette thématique.

8 La troisième raison est qu'une partie importante des écrivains soudanais vivent à l'étranger (dans les pays du Golfe, en Égypte, en Europe ou en Amérique du Nord) et que, par conséquent, lorsqu'ils abordent dans leurs livres la problématique de l'exil, ils décrivent des situations qu'ils ont vécues directement ou bien dont ils ont été les témoins.

\section{Une terre d'exil moins connue : les pays communistes}

9 Si l'influence de Saison d'une migration vers le nord a été, on l'a dit, considérable sur la littérature soudanaise, la manière d'aborder l'émigration a bien sûr fortement évolué en quarante ans. Tout d'abord, les causes de l'émigration se sont diversifiées - alors que le héros du roman d'Al-Tayyib Salîh se rend en Europe pour étudier, les personnages d'Ahmad Al-Malik ou d'Amir Tagelsir', par exemple, tentent de quitter le Soudan pour des motifs politiques ou économiques. Les destinations ont changé elles aussi : Mustafa Sa îd se rendait tout naturellement à Londres, capitale de l'ancienne puissance colonisatrice, alors que les personnages des nouvelles et des romans plus récents s'installent ailleurs en Europe (aux Pays-Bas, en Suisse, en Autriche), voire même aux États-Unis.

10 Ali, le personnage de la nouvelle de Badri intitulée "Le Politicien ${ }^{9}$, choisit une destination a priori bien plus exotique: la Tchécoslovaquie. En effet, Ali est un jeune communiste soudanais exalté qui obtient une bourse pour aller étudier à l'Université de Prague, puis à celle de Bratislava. Aucune date n'est mentionnée, mais l'on sait qu'il est 
encore à l'école secondaire lors de l'accession de son pays à l'indépendance, en 1956, qu'il suit des événements politiques tels que la mort de Patrice Lumumba en 1961, la construction du haut-barrage d'Assouan dans les années 1960 et l'alliance du général Ibrahim Abboud (au pouvoir entre 1958 et 1964) avec les Américains avant son départ et qu'il est de retour au Soudan lors de la chute du président soudanais Gaafar Nimeiri, en 1985. Ali se rend donc vraisemblablement à Prague dans les années 1960, probablement avant 1968, puisqu'il ne fait aucune allusion au "printemps de Prague », un événement difficile à passer sous silence. La nouvelle comble ainsi un pan important, et pourtant très peu abordé, de l'histoire de l'émigration temporaire dans le monde arabe et, plus largement, dans le Tiers-Monde: le parcours des étudiants arabes, africains et sudaméricains qui se rendaient en Union soviétique et dans les autres pays communistes pour accomplir leurs études. Il n'est pas rare de rencontrer, en Syrie, au Soudan, au Yémen ou ailleurs des ingénieurs et des médecins qui parlent le russe, le tchèque ou d'autres langues slaves (mais aussi le roumain), ayant obtenu dans leur jeune temps une bourse pour étudier dans une université d'un pays communiste. Cette politique éducative visant à former ses élites en URSS et dans les autres pays de l'Est était particulièrement développée dans les pays ayant ouvertement adopté le socialisme comme système politique à partir des années 1960, au lendemain de la vague des indépendances - la Syrie dès les années 1960 et la république populaire du Yémen en 1970 pour les pays arabes, la Tanzanie de Julius Nyerere à partir de 1967 et l'Angola du MPLA en 1975 pour l'Afrique...

11 Plusieurs auteurs arabes ont eu l'occasion d'étudier dans les pays de l'Est, à Moscou comme l'Irakien Ghaib Tuma Firman et le Libyen Ibrahim Al-Kuni - qui a étudié la littérature à l'Institut Maxime Gorki -, en Pologne comme le Soudanais Rauf Mus'ad, en Tchécoslovaquie comme Shawgi Badri, mais aussi en Bulgarie et ailleurs. Certains se sont d'ailleurs inspirés de leurs séjours estudiantins pour rédiger une partie de leur œuvre, comme par exemple le Libanais Nizar Dandash ${ }^{10}$.

12 Le soutien offert aux pays arabes et africains, qui entrait dans un volet de politique internationale théorisé par le Parti communiste soviétique de 1960, dans sa déclaration de Moscou, ne se limitait toutefois pas à l'accueil d'étudiants venus du Tiers-Monde : des ingénieurs, des enseignants, des médecins et d'autres spécialistes russes, tchèques, polonais et autres furent également envoyés sur place, de la Somalie à la Guinée en passant par l'Égypte et l'Éthiopie. Le cas du Soudan est particulier, puisque les étudiants candidats au départ qui sont décrits dans la nouvelle de Shawgi Badri ne bénéficiaient pas de bourses du gouvernement, mais étaient choisis par le Parti communiste soudanais, qui était très populaire à l'époque ${ }^{11}$.

Pour en revenir à Ali, son parcours est donc très différent de celui des personnages d'autres romans soudanais abordant le thème de l'émigration: le narrateur idéalise le monde extérieur, certes, mais à contre-courant de ses compatriotes. Alors que ces derniers imaginent trouver une vie meilleure à Londres, à Paris ou à Amsterdam, lui est persuadé que l'herbe est plus verte en Union soviétique.

Par ailleurs, alors que le héros de la nouvelle « Un garçon de l'extrême sud » d'Ahmad AlMalik ${ }^{12}$ fut forcé de quitter son pays et de se rendre aux Pays-Bas pour des raisons politiques, Ali choisit au contraire de quitter sa patrie pour ces mêmes raisons : ce n'est pas son opposition au gouvernement en place qui le pousse à partir, mais l'idéalisation d'un système politique. «Parti» (il faut comprendre le Parti communiste soudanais) car Ali aurait préféré se 
rendre à Moscou, capitale de la vraie patrie du communisme, et non à Prague ou dans une autre ville jugée « secondaire $»^{13}$.

\section{Le racisme}

Mais Ali est vite déçu par la réalité qu'il découvre: tout d'abord, il est rapidement confronté tantôt à l'indifférence, tantôt au racisme, loin des rapports humanistes et fraternels décrits dans la littérature officielle: dès l'aéroport, les gens semblent se moquer de lui et des autres étudiants africains. Quelques jours plus tard, il se fait tabasser par un groupe de jeunes Tchèques avec qui il veut discuter de politique. Mais l'événement décisif sera le refus de le laisser entrer, lui et d'autres collègues africains, dans une discothèque praguoise :

Lors d'une réunion avec le président de l'organisation culturelle à Prague, dans son imposant bureau des studios Brandov, les Africains demandèrent pourquoi ils n'avaient pas le droit d'aller au dancing "La lanterne » ou même dans les autres dancings. Le responsable et membre du Parti répondit que c'était parce que quelques étudiants congolais y avaient causé des problèmes. Alors Ali lui demanda pourquoi ils n'avaient pas interdit l'entrée aux étudiants congolais uniquement. Le responsable culturel répondit que «le Congo était grand, il y avait là-bas le Nigeria, le Ghana, l'Afrique et bien d'autres états encore, ils ne pouvaient donc pas faire le tri entre tous ces Congolais $\aleph^{14}$.

La dénonciation du racisme dans cette nouvelle de Badri est intéressante à plusieurs égards. D'une part, le racisme est souvent associé dans la littérature arabe à l'expérience qu'en ont des immigrés arabes en Europe occidentale ou aux États-Unis. Ici, le héros est confronté au racisme dans un monde où, précisément, il ne devrait pas en avoir, étant donné le système politique égalitaire censé y être en vigueur. Nous verrons d'ailleurs plus loin que dans un premier temps, le héros niera ou minimisera le phénomène.

Mais l'auteur va plus loin, puisqu'il dénonce aussi le racisme dont sont victimes les Soudanais de la part des autres Arabes, qui semblent les considérer comme des Africains et non comme Arabes au même titre qu'eux-mêmes, Égyptiens, Syriens ou Palestiniens. Rappelons que le Soudan est un pays multiculturel dans lequel se côtoient des populations africaines et arabes, musulmanes, animistes et chrétiennes, mais que la composante arabo-musulmane, présente depuis des siècles dans le nord du pays, y domine largement l'espace culturel et politique. Le premier épisode se déroule au Caire : pour se rendre à Prague, Ali doit y passer pour y obtenir son visa et acheter son billet d'avion. Durant son séjour dans la capitale égyptienne, il va faire la connaissance d'un groupe de Palestiniens. Le conflit israélo-palestinien occupe une place fondamentale dans la conscience politique arabe contemporaine, au point d'éclipser souvent bien d'autres causes dignes d'intérêt dans cette partie du monde, ce dont se moque gentiment l'auteur à travers la fascination d'Ali pour ses collègues palestiniens. En effet, il décide d'organiser une fête en leur honneur, fier de pouvoir rencontrer des «combattants» de la cause palestinienne en chair et en os, et se coupe en quatre pour eux. Alors qu'il part préparer le thé dans la cuisine, il surprend la conversation de deux d'entre eux, dont l'un dit: «C'est quoi cette mascarade? Qu'est-ce que ces nègres ont à voir avec la Cause ? Ils m'ont vraiment donné mal de tête $! »^{15}$ Survient alors une descente de police, durant laquelle Ali semble recevoir plus de coups que les autres. Apparemment, le héros fait l'amère expérience du racisme dans le monde arabe en raison de la couleur de sa peau, à la fois à travers les paroles des étudiants palestiniens et les coups des policiers égyptiens... 
L'épisode se reproduira ensuite en Tchéquie, à l'université de Prague : « Les relations avec les étudiants arabes n'étaient pas très bonnes, et de nombreux Soudanais furent étonnés de découvrir que certains étudiants arabes ne les considéraient pas du tout comme des frères. $\aleph^{16}$ La question de ce déséquilibre entre l'identité arabe des Soudanais telle qu'elle est ressentie par eux-mêmes et la perception qu'en ont les autres Arabes, qui les considèrent comme des Africains, a été abordée par d'autres auteurs, notamment Jamâl Muhammad Ibrâhîm dans son roman Nuqtat al-talâshî (Le Temps de consomption) ${ }^{17}$.

19 Finalement, l'auteur démontre habilement que le racisme est hélas une donnée universelle, présente en Europe occidentale, mais aussi en Europe centrale... et dans le monde arabe !

\section{Les républiques socialistes, un monde parfait}

20 Les pays socialistes sont idéalisés par le pauvre Ali, persuadé avant d'entreprendre son voyage « qu'en Union soviétique, même à la loterie il n'y a jamais de perdant, quiconque achète un billet gagne quelque chose $»^{18}$. Un tel idéalisme le poussera à nier, à minimiser ou à excuser dans un premier temps les incidents plus ou moins graves qui ternissent sa confrontation avec la réalité, dès son arrivée à l'aéroport de Prague. Ainsi, lorsqu'il est confronté aux premières manifestations du racisme dans la capitale tchèque, il soutient "qu'il n'y a pas de discrimination raciale dans les états socialistes, parce que ce phénomène existe uniquement dans le système capitaliste, en raison de l'exploitation ${ }^{19}$. Il lui faudra vivre lui-même plusieurs expériences malheureuses pour accepter les arguments de ses amis qui considèrent que le « racisme n'a pas uniquement un aspect économique, il y a aussi le background culturel, psychologique... $»^{20}$

21 Parallèlement, les gens qui naissent et vivent dans les pays socialistes ne partagent pas forcément le même enthousiasme que ceux qui, comme Ali, ont fait le choix d'y vivre, d'où la réflexion des jeunes Tchèques «aux longs cheveux» qui tabassent le héros lorsqu'il tente de parler politique avec eux: "N'a-t-on pas assez à faire avec nos communistes, pour que maintenant on voie même arriver des communistes noirs? $»^{21} \mathrm{Il}$ est reçu de la même manière par un groupe d'étudiants vietnamiens à qui il parle avec enthousiasme « de la défaite française, du général Giap, du président Ho Chi Min et de la solidarité internationale... $»^{22}$

Mais Ali sera confronté encore à bien d'autres déconvenues: sa découverte de la corruption à tous les échelons, notamment pour obtenir son diplôme universitaire (ce qui lui causera de sérieux problèmes avec ses professeurs, qui n'apprécient guère sa franchise), le matérialisme du citoyen lambda et aussi les inimitiés entre Tchèques et Russes («les Tchèques détestent plus les Russes qui les ont pourtant libérés que les Allemands qui les ont occupés ", observe-t-il ${ }^{23}$ ), loin de sa propre vision naïve de l'amitié entre les peuples et de la solidarité internationale.

En ce sens, l'expérience du narrateur rejoint en de nombreux points les témoignages d'Occidentaux ayant fait, eux aussi, le voyage de l'autre côté du rideau de fer, pensons notamment au livre-témoignage de Jean et Nina Kéhayan, Rue du prolétaire rouge ${ }^{24}$ : pleins d'enthousiasme avant le départ, la réalité à laquelle ils sont rapidement confrontés en Union soviétique les pousse, après une période de négation de la réalité, à revoir entièrement leurs positions, au point d'être rejetés par leurs compagnons à leur retour en France... 


\section{L'Europe centrale dans les autres nouvelles de Badri} revue les autres communautés étrangères que la Suède a accueillies à travers son Histoire, y compris les Finlandais, les Grecs, les Italiens ou même les Américains opposés à la guerre du Vietnam dès les années $1960^{29}$. Ainsi, l'identité slave de Zadravko n'a pas d'importance en soi, le personnage n'est en réalité qu'un représentant parmi d'autres de la multiculturalité des villes européennes d'aujourd'hui, qui peut être slave, mais aussi africaine comme le second protagoniste de la nouvelle. On retrouve cette façon de souligner ce brassage culturel chez d'autres auteurs soudanais installés en Europe, comme Ahmad Al-Malik dans sa nouvelle « Al-hubb wa-l-vudkâ » (L'Amour et la Vodka), mais aussi, plus largement, chez d'autres auteurs arabophones comme l'Irakien Samuîl Shim'ûn dans son roman 'Irâqî fî Bârîs (Un Irakien à Paris) ou le Marocain Abdelmounem Chentouf dans sa nouvelle "Taraf al-yawm al-akhîr » (L'Extrémité du dernier jour) ${ }^{30}$, lorsqu'ils écrivent à propos de villes comme Amsterdam, Paris ou Bruxelles. Tous mettent en scène, à dessein, des personnages venus des quatre coins du monde, qu'ils soient Arabes, Africains, Asiatiques ou originaires d'Europe orientale.

\section{Le voyage d'Ali en Tchécoslovaquie : fiction ou réalité?}

Vraisemblablement, de nombreux lecteurs ont considéré que cette nouvelle constituait un récit largement autobiographique. Du coup, l'auteur s'en est défendu en 2007 dans un article intitulé «Les communistes qui m’ont arrêté et chassé de Tchécoslovaquie » ${ }^{31}$. On y découvre une série de détails de la vie de l'auteur que l'on peut mettre aisément en relation avec la nouvelle «Le Politicien». Badri a effectivement étudié à Prague, en compagnie d'autres étudiants arabes et africains, entouré de nombreux communistes soudanais. Il a rencontré des problèmes avec ses professeurs, au point d'être finalement 
chassé du pays. Il parle aussi dans cet article du matérialisme du citoyen tchèque ordinaire de l'époque, de la discrimination envers les étudiants africains, et même d'une bagarre entre étudiants soudanais dans la discothèque "La lanterne» de Prague, un événement similaire à celui mentionné dans « Le Politicien ». Par contre, l'auteur insiste aussi sur le fait qu'il n'a jamais été membre du Parti communiste soudanais (mais il se positionne clairement en tant que socialiste), qu'il critique d'ailleurs très sévèrement le Parti communiste soudanais, précisant que « les communistes soudanais refusaient toute critique du système ou de la société tchèque » (on pense bien sûr aux réactions d'Ali dans les premiers temps de son séjour à Prague) ou encore que "de nombreux diplômés [soudanais] de Tchécoslovaquie n'auraient jamais réussi leurs études en raison de leur faiblesse académique, s'ils n'avaient pas été membres du Parti communiste soudanais ». Badri semble donc avoir mêlé dans cette nouvelle son propre vécu et celui de ses compatriotes parmi les étudiants de l'époque, qu'ils soient ou non communistes.

\section{Conclusion}

28 À travers la lecture du "Politicien", on en apprend finalement peu de choses sur la perception qu'a l'auteur de la Tchécoslovaquie, où il a pourtant vécu plusieurs années. En réalité, la dimension politique de la nouvelle occulte largement la dimension culturelle, Prague n'est en définitive que le décor nécessaire pour aborder le travail de remise en question d'un militant communiste qui, aveuglé par son enthousiasme, aura besoin d'un peu de temps pour accepter la réalité. Ali se rend dans un pays communiste avant de se rendre dans un pays slave - s'il avait pu obtenir une bourse pour la Roumanie ou pour Cuba, il l'aurait sans doute acceptée, et il aurait subi les mêmes déconvenues. À cet égard, la joie démesurée que ressent Ali lorsqu'il rencontre pour la première fois de sa vie un couple de Russes ${ }^{32}$ est particulièrement significative: encore une fois, ce n'est pas la culture russe qui l'attire, il est tout simplement heureux d'enfin se retrouver face à des représentants de la nation communiste par excellence, plutôt que de simples camarades de pays satellites.

Il n'empêche qu'en quelques pages, l'auteur est parvenu à aborder deux questions essentielles de manière originale : la question de l'idéalisme politique et de ses inévitables désillusions, et celle de l'identité - récurrente dans la littérature soudanaise.

\section{NOTES}

1. Badri Sh., Al-mushbak[La Pince à linge], Dâr Al-'Âlamiyya, Le Caire, 2003, 156 p.

2. Badri Sh., Hakâwî Umdurmân [Le Conteur d'Omdourman], Dâr Al-'Âlamiyya, Le Caire, 2008, $269 \mathrm{p}$.

3. Muhammad Ahmad Al-Mahdi (1844-1885) était une personnalité à la fois religieuse et politique, prétendant être envoyé par Dieu. Il initia une rébellion de grande envergure contre l'occupant colonial britannique et parvint à arracher Khartoum au général Gordon, en 1885. Il fonda alors un état d'inspiration théocratique, dont Omdourman, ville voisine de Khartoum, 
devint la capitale. Après la mort d'Al-Mahdi, son mouvement insurrectionnel perdura jusqu'en 1898, date de la victoire finale des Britanniques. Voir notamment Holt P. M. \& Daly M. W, A History of the Sudan (Une histoire du Soudan), Longman, New York et Londres, 1994 (1961), p. 5.

4. Al-Tayyib S., Mawsim al-hijra ilâ al-shamâl (Saison d'une migration vers le nord), Le Caire, 1968, $171 \mathrm{p}$.

5. Voir notamment Takieddine Amyuni M., Tayeb Salih's Season of a Migration to the North: a Casebook [Saison d'une migration vers le nord de Tayeb Salih: Recueil d'articles], American University of Beirut, Beyrouth, 1985, 173 p.

6. Pour un aperçu de la littérature soudanaise, voir Luffin X., "La littérature soudanaise aujourd'hui ", in : Études littéraires africaines. Littératures du Soudan, t. XXVIII, APELA, Paris, 2010, pp. 12-15.

7. Al-Hakîm T., 'Usfûr min al-sharq [Un oiseau d'Orient], Dâr al-kitâb al-lubnânî, Beyrouth, 1980, 192.

8. Al-Malik A., "Sabî min aqsâ al-janûb » [Un jeune homme de l'extrême sud], in : Nûra dhât aldafầir [Nora aux cheveux tressés], Dâr 'Azza, Le Caire, 2006, 175 p. ; Tagelsir A., Le parfum français, L'Harmattan, Paris, 2010, 145 p.

9. Badri Sh., « Al-siyâsî » [Le Politicien], in : Al-mushbak, Op. cit., pp. 110-120.

10. Nizar Dandash, Yawmiyyât Mûskû al-khadrâ' (Souvenirs de Moscou la verte), Al-dâr al-'arabiyya li-1-'ulûm, Beyrouth, 2002, 231 p.

11. Pour le cas particulier des étudiants africains en Tchécoslovaquie et de la participation tchécoslovaque aux infrastructures des pays africains récemment indépendants, voir notamment Beck C. F., «Czechoslovakia's Penetration of Africa 1955-1962 » [la pénétration en Afrique de la Tchécoslovaquie, 1955-1962], in : World Politics, t. XV, nº 3, avril 1963, pp. 403-416.

12. Al-Malik A., Op. cit., p. 152.

13. Badri Sh., « Al-siyâsî », Op. cit., p. 112.

14. Ibid., p. 115.

15. Ibid.. p. 113.

16. Ibid., p. 116.

17. Muhammad Ibrâhîm J., Nuqtat al-talâshî [Le point d'évanescence], Dâr al-sâqî, Beyrouth, 2007, $143 \mathrm{p}$.

18. Badri Sh., «Al-siyâsî », Op. cit., p. 111.

19. Ibid., p. 114.

20. Idem.

21. Idem.

22. Idem.

23. Idem.

24. Kéhayan J. \& N., Rue du prolétaire rouge, Le seuil, Paris, 1978, 221 p.

25. Badri Sh., "Al-siyâsî », Op. cit., pp. 16-21.

26. Ibid., p. 19.

27. Idem.

28. Ibid., p. 17.

29. Idem.

30. Al-Malik A., " al-hubb wa-l-vûdkâ » [L'Amour et la Vodka], in : Nûra dhât al-dafâ'ir [Nora aux cheveux tressés], Le Caire, Dâr 'Azza, 2006, 175 p. ; Shim'ûn S., 'Irâqî fî Bârîs [Un Irakien à Paris], Al-jamal, Beyrouth, 2008, 349 p. ; Chentouf A., "Taraf yawm al-akhîr » [L'Extrémité du dernier jour], in : Al-khurûj min al-sulâla [Sortir de la famille], Wizârat al-thaqâfa, Rabat, 2009, 71 p.

31. URL : http ://sbadri.maktooblog.com/page/2/ (consulté le 15/09/2010).

32. Badri Sh., « Al-siyâsî », Op. cit., p. 116. 


\section{RÉSUMÉS}

Shawgi Badri est un écrivain soudanais installé depuis plusieurs décennies en Suède. Il est l'auteur de deux recueils de nouvelles, Al-mushbak (La Pince à linge) et Hakâwî Umdurmân (Le Conteur d'Omdourman), dans lesquels il s'inspire de ses souvenirs de jeunesse dans sa ville d'origine, Omdourman, mais aussi de son passé d'étudiant politisé et engagé. L'une de ces nouvelles, «Le Politicien », retrace les illusions et les désillusions d'un jeune étudiant soudanais, attiré par le communisme, qui part étudier en Tchécoslovaquie dans les années 1960.

Shawgi Badri is a Sudanese author who has been living in Sweden for several decades. He wrote two collections of short stories, Al-mushbak ("The Clothes Peg") and Hakâwî Umdurmân ("The Storyteller of Omdurman"). He draws inspiration from his youth, during which he still used to live in Omdurman, as well as from his experience as a student involved in politics. One of his short stories, "The Politician", depicts the dreams and disappointments of a young Sudanese student, attracted by communism, who gets a scholarship to study in Czechoslovakia during the sixties.

INDEX

Index chronologique : communisme, XXe siècle Index géographique : Soudan, Tchécoslovaquie

Mots-clés : exil, racisme

\section{AUTEURS}

\section{XAVIER LUFFIN}

chargé de cours (Maître de conférences) à l'Université Libre de Bruxelles 